\title{
Quantitative automated circumpapillary microvascular density measurements: a new angioOCT-based methodology
}

\author{
Danilo A. Jesus $\mathbb{1}^{1,2}$ • João Barbosa Breda $\mathbb{1}^{1,3,4} \cdot$ Karel Van Keer ${ }^{1,5}$. Amândio Rocha Sousa ${ }^{3,4}$. \\ Luis Abegão Pinto $\mathbb{1}^{6} \cdot$ Ingeborg Stalmans ${ }^{1,5}$
}

Received: 6 April 2018 / Revised: 6 August 2018 / Accepted: 16 August 2018 / Published online: 11 September 2018

(c) The Royal College of Ophthalmologists 2018

\begin{abstract}
Purpose To develop a new methodology to detect glaucoma damage based on circumpapillary microvascular density (cpmVD) as measured by optical coherence tomography angiography (angioOCT).

Methods AngioOCT scans from a random eye of 40 healthy subjects (aged $63 \pm 14$ years) and 82 glaucoma patients (aged $66 \pm 9$ years with an average visual field loss of $-7.8 \pm 6.5 \mathrm{~dB}$ ) were used to develop a new angioOCT evaluation methodology. Optic disc-centred $3 \times 3 \mathrm{~mm}$ images were collected (Angioplex ${ }^{\circ}$, Zeiss Cirrus 5000 HD-OCT). An annular area with an inner radius of $1.16 \mathrm{~mm}$ and outer radius of $1.44 \mathrm{~mm}$ was chosen as the region of interest (ROI), with cpmVD calculated through lower envelope modulation avoiding the influence of the major retinal vessels. Additionally, the cpmVD and the microvascular density (mVD), stratified by sectors, were compared with the respective retinal nerve fibre layer (RNFL) thickness.

Results A significantly lower cpmVD was observed in the glaucoma group, for all visual field sectors $(p<0.001)$, when comparing to the healthy group. The inferior and superior mVD sectors showed the largest differences between groups. Moreover, glaucoma was linked to a reduction of cpmVD variation when compared to the healthy group. Lastly, a comparison between two healthy subgroups showed that the method is not biased by the presence of slight movement artefacts, optic disc dimensions and vessel crowding $(p>0.05)$.

Conclusions The presented open-source methodology provides a robust quantitative analysis of the $360^{\circ} \mathrm{mVD}$. It shows that cpmVD, and mVD sectors measured by angioOCT, can be used in everyday glaucoma practice.
\end{abstract}

These authors contributed equally: Danilo A. Jesus and João Barbosa Breda

Electronic supplementary material The online version of this article (https://doi.org/10.1038/s41433-018-0207-z) contains supplementary material, which is available to authorized users.

Danilo A. Jesus

danilo.ajesus@kuleuven.be

1 Department of Neurosciences, Research Group of Ophthalmology, KU Leuven, Leuven, Belgium

2 Departments of Radiology and Nuclear Medicine and Medical Informatics, Biomedical Imaging Group Rotterdam, University Medical Center Rotterdam, Rotterdam, The Netherlands

3 Faculty of Medicine, University of Porto, Porto, Portugal

4 Department of Ophthalmology, Centro Hospitalar São João, Porto, Portugal

5 Department of Ophthalmology, University Hospitals Leuven, Leuven, Belgium

6 Department of Ophthalmology, Centro Hospitalar Lisboa Norte, Lisboa, Portugal

\section{Introduction}

Glaucoma is the leading cause of irreversible blindness in the world and its prevalence will increase in the following decades [1, 2]. A progressive loss of retinal ganglion cells (RGCs) is the hallmark behind the disease and several insults can lead to this progressive damage: from a high intraocular pressure (considered the main risk factor) to impaired nutrition and vascular dysfunction [3-7]. A number of studies have shown that irrespective of the type of glaucoma, the loss of RGCs is accompanied by an attenuation of retinal microvasculature [8-11]. Which of the two happens first remains to be answered. Optical coherence tomography (OCT) angiography (angioOCT) has recently emerged as an imaging technique to quantify the retinal microvasculature in a non-invasive, fast and reproducible way. AngioOCT employs motion contrast imaging to high-resolution volumetric blood flow information generating new angiographic data that complements traditional 
Fig. 1 AngioOCT image of a healthy (left) and a glaucoma (right) subject
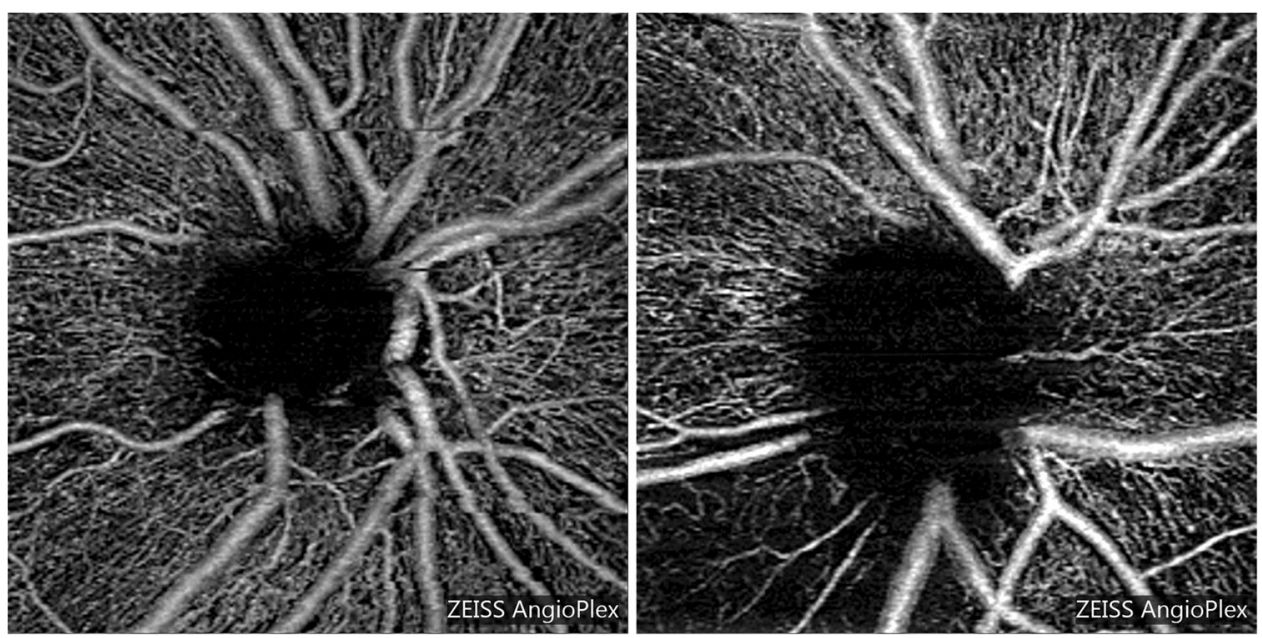

OCT images. Although several angioOCT devices are available, only a few provide quantitative circumpapillary microvascular density (cpmVD) analysis in their commercially available software [10, 12-14]. These measurements are calculated based on the percentage area occupied by all vessels present in the peripapillary region, including microvasculature and large retinal vessels. Such analysis leads to measurement bias since the large vessel profile differs between individuals and does not provide data about the perfusion of the optic nerve head (ONH). Additionally, the current methods do not correct images for the fovea-disc axis (which influences sectorial measurements). This heterogeneity in measurement methods precludes an accurate comparison of the studies that have been conducted. Therefore, the aim of this study was to develop an innovative opensource tool that extracts quantitative cpmVD values from angioOCT scans, while correcting the images for the foveadisc axis and avoiding the influence of large retinal vessels.

\section{Methods}

\section{Subjects and image acquisition}

Forty healthy subjects (aged $63 \pm 14$ years) and 82 openangle glaucoma patients (aged $66 \pm 9$ years with an average visual field mean deviation of $-7.8 \pm 6.5 \mathrm{~dB}$ ) were recruited from the Leuven Eye Study cohort [7]. This is one of the largest studies conducted in glaucoma patients with the primary purpose of studying vascular parameters. In short, healthy subjects were recruited from those accompanying glaucoma patients (excluding blood relatives) and were screened by a glaucoma specialist (I.S.). Those with a family history of glaucoma, rim thinning or notching, asymmetrical cup/disc ratio or any other optic disc structural change such as disc haemorrhage, or an IOP above $21 \mathrm{mmHg}$ were excluded. Patients with open-angle glaucoma were consecutively included and the diagnosis was defined as having characteristic optic disc damage and visual field loss [15]. Patients with diabetes mellitus were excluded, since this pathology is a known confounder in vascular-related research. Additionally, patients were excluded if they had a history of ocular trauma or any other eye disease (including high ametropias, defined as hyperopia $>4$ dioptres and myopia $>6$ dioptres). The eye with greater glaucomatous damage was selected for the study. This study was approved by the Institutional Review Board of the University Hospitals Leuven and adhered to the tenets of the Declaration of Helsinki. All patients signed an informed consent prior to the study evaluation. For each patient, a $3 \times 3 \mathrm{~mm}$ optic disc-centred angiography scan was acquired (via undilated pupil), and the superficial layer scan (which corresponds to the radial peripapillary capillaries layer) exported (Cirrus $5000 \mathrm{HD}^{\mathrm{OCT}}{ }^{\oplus}$, Angioplex ${ }^{\circledR}$, Carl Zeiss, Dublin, USA; 10.0 software version) (Fig. 1). Poorquality images, which were defined as having a quality score less than six or identifiable floaters were excluded from the analysis. PanoMap images were also exported to correct cpmVD measurements for the fovea-disc axis. Additionally, the retinal nerve fibre layer (RNFL) thickness was measured with the same device following the angiography scan, and average and sectorial (temporal, superior, nasal and inferior) values were exported. All acquisitions were conducted by the same person (J.B.B.).

\section{Image processing}

The different steps employed in image processing are summarized in Fig. 2. AngioOCT images were corrected (rotated) for the fovea-disc axis, using the method presented by Mwanza et al. [16]. In this method, PanoMap images are used to calculate the angle between the fovea and the optic disc, through the relative positions of the optic disc and macular centres, aligning this axis with a horizontal line 
(A)

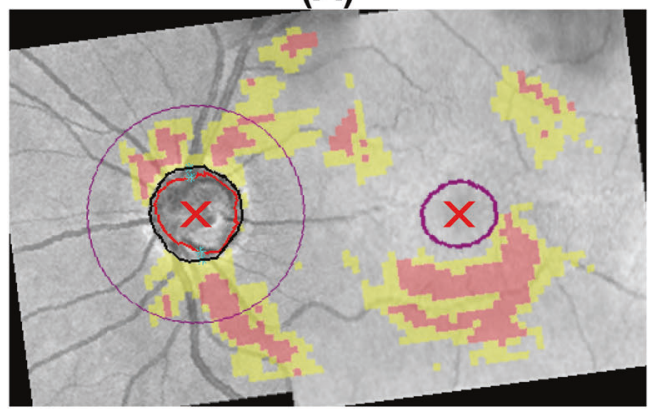

(B)

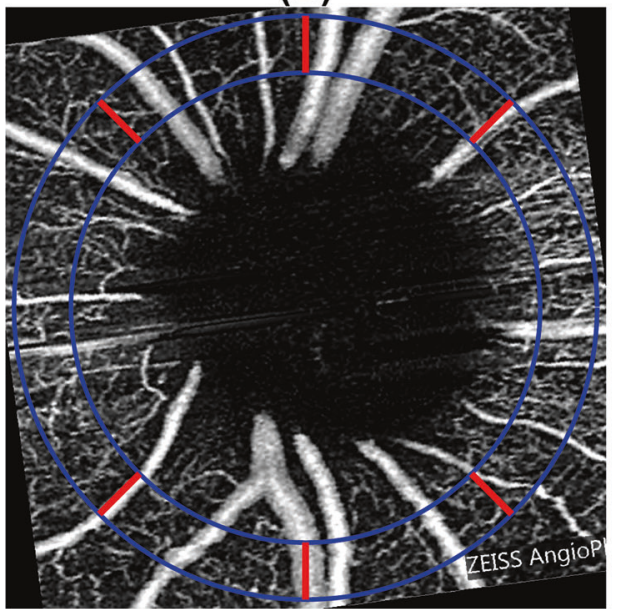

Fig. 2 a PanoMap image rotated $\left(7.6^{\circ}\right)$ to align the fovea-disc axis with $0^{\circ}$. b AngioOCT image corrected (rotated) for the fovea-disc axis with the respective ROI delineated by the blue lines. Red lines

$\left(0^{\circ}\right)$. After fovea-disc axis correction, an annular area with an inner radius of $1.16 \mathrm{~mm}$ and outer radius of $1.44 \mathrm{~mm}$ was chosen as the region of interest (ROI). The outer diameter is the largest possible without capturing any trademark symbols (present on the lower right corner), while the inner diameter was selected to maximize the width of the ROI, without reaching the optically hollow $\mathrm{ONH}$ area. The largest hollow area among all subjects was selected to ascertain the default inner radius. In order to easily visualize and manipulate the data, the annular ROI was converted to a horizontal linear ROI $(92 \times 1080$ pixels $)$ composed by the meridional lines around the $\mathrm{ONH}$ centre with a $1 / 3^{\circ}$ rotation resolution. A row vector $(1 \times 1080)$ containing the mean value of each column was obtained and filtered with a thirdorder median filter. Lastly, the lower peak envelope of the signal using a spline over local maxima separated by 37 pixels $\left(\sim 12.3^{\circ}\right.$ in the circular ROI) was estimated as true cpmVD. The cpmVD was plotted starting at $0^{\circ}$ at the temporal side (landmark) and then following the temporal-superior-nasal-inferior-temporal direction. This allows a visual comparison with the standard mean RNFL plots. Six sectors were defined to allow for a
(C)

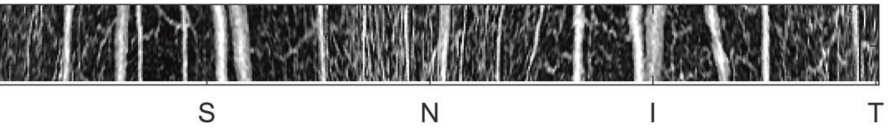

(D)



(E)

delineate six sectors. c Horizontal linear ROI. d Averaged signal (black) obtained from $\mathbf{c}$ and the lower peak envelope (red) of the filtered signal. e Boxplot of the cpmVD according to six sectors

structure-structure correlation between $\mathrm{mVD}$ and mean RNFL thickness. Briefly, the nasal and temporal sectors have $90^{\circ}$ each, while the superior and inferior sectors are both divided in 2 sectors of $45^{\circ}$ (Fig. 2b-2e). All microvascular density units are expressed as arbitrary units (a.u.). Unlike the majority of the methods described in the literature, our procedure is not based on a white pixel ratio over a denominated region, and therefore, the results are not presented as white pixel percentage. Since the obtained microvascular density is algorithm-dependent, resulting from the radial lower envelope of the normalized image intensity, it is more appropriated to express the obtained microvascular density as arbitrary units. This software available online [17] as well as the graphs and statistical analyses were developed with Matlab (Mathworks, Natick, MA). An introductory manual can be found as a supplementary file.

\section{Sources of bias}

All images of healthy subjects were graded by two experts (J.B.B. and I.S.) for the presence of minor movement artefacts, large or small ONH optically hollow areas, and 
high or low large vessel crowding. A blind group comparison was done to assess if these three features influenced the measured cpmVD. Only images from healthy subjects were used since the purpose of this analysis was to isolate the true effect of image artefacts from that of pathologic changes. Additionally, the method's repeatability was assessed in one control eye taking a set of 10 images obtained sequentially during a single visit. The variability was measured by the coefficient of variation, calculated as the standard deviation divided by the mean of the measured values.

\section{Statistical analysis}

After assessing the non-normal distribution (Kolmogorov-Smirnov test) of cpmVD, the non-parametric Wilcoxon rank-sum test with 0.05 level of significance was used to test the statistical differences between the healthy and the glaucoma groups, whereas the Kruskal-Wallis test was used to assess the differences between sectors within each group. Lastly, the Spearman rank correlation was used to measure the association between the mVD and the RNFL thickness (mVD uncorrected for fovea-disc axis, since the RNFL sectors are automatically provided with no correction for such axis). The ability of $\mathrm{mVD}$ and RFNL thickness to discriminate between controls and glaucoma subjects was assessed by measuring the area under the receiver operating characteristic curve (AROC) with a 95\% confidence interval.

\section{Results}

No significant age difference existed between the healthy and the glaucoma groups $(p=0.143)$. The coefficient of variation for 10 images in one control eye obtained sequentially during a single visit was $3.6 \%$ for the standard ROI width used in this study $(0.28 \mathrm{~mm})$. The coefficient of variation decreased to $2.9 \%$ when the ROI width was duplicated $(0.56 \mathrm{~mm})$ showing the precision increase for larger ROI widths. The healthy subjects presented a higher cpmVD when compared to glaucoma patients (Fig. 3). Moreover, the healthy control group showed a high cpmVD variation, with a higher density in the superior and inferior areas. Such variation seems to decrease with glaucomatous damage since glaucoma patients presented a flatter, more uniform, microvascular density profile. Regarding the sectorial analysis, the greatest cpmVD disparity between groups was found in the inferior and supero-temporal sectors.

The correlation between the peripapillary microvascular density, non-corrected for the fovea-disc axis, and the RNFL thickness for the healthy and the glaucoma groups, is shown in Table 1. Only the superior and temporal sectors presented a significant correlation in the healthy group, whereas in the glaucoma group, significant correlations

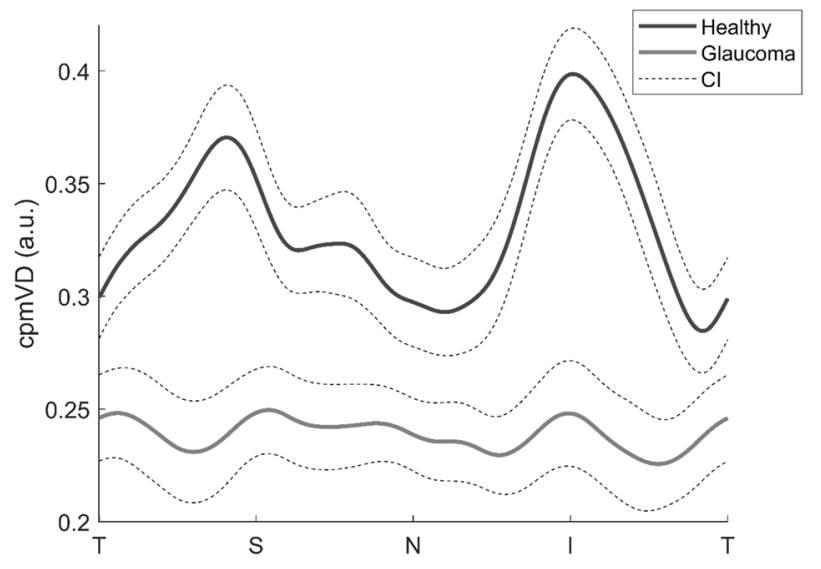

\begin{tabular}{c|c|c|c}
\multirow{2}{*}{ mVD } & Healthy & Glaucoma & \multirow{2}{*}{ AROC (CI) } \\
\cline { 2 - 3 } & Median (IQR) & Median (IQR) & \\
\hline cp & $0.34(0.06)$ & $0.24(0.10)$ & $0.89(0.83$ to 0.94$)$ \\
\hline T & $0.30(0.08)$ & $0.23(0.10)$ & $0.77(0.69$ to 0.86$)$ \\
\hline ST & $0.36(0.10)$ & $0.23(0.13)$ & $0.85(0.79$ to 0.92$)$ \\
\hline SN & $0.34(0.08)$ & $0.24(0.14)$ & $0.79(0.71$ to 0.87$)$ \\
\hline N & $0.30(0.07)$ & $0.24(0.09)$ & $0.78(0.70$ to 0.86$)$ \\
\hline IN & $0.36(0.10)$ & $0.23(0.14)$ & $0.86(0.80$ to 0.93$)$ \\
\hline IT & $0.39(0.09)$ & $0.22(0.13)$ & $0.87(0.80$ to 0.93$)$
\end{tabular}

Fig. 3 Averaged circumpapillary microvascular density (cpmVD) of the healthy control and glaucoma groups delineated by the blue and the red lines, respectively. The dashed black lines denote the $95 \%$ confidence intervals. All variables described as median; interquartile range (IQR); area under the receiver operating characteristic curve (AROC); 95\% confidence Interval (CI); microvascular density (mVD); circumpapillary (cp); temporal (T); supero-temporal (ST); supero-nasal (SN); nasal (N); infero-nasal (IN); infero-nasal (IT); p-value $<0.001$ for all comparisons.

Table 1 Comparison between the peripapillary microvascular density and the RNFL thickness for the healthy and the glaucoma groups (noncorrected for the fovea-disc axis)

\begin{tabular}{lllll}
\hline & $\mathrm{mVD}$ & $\mathrm{RNFL}$ & $\rho$ & $p$-value \\
\hline Healthy & & & & \\
$\mathrm{cp}$ & $0.34(0.06)$ & $90(8)$ & 0.04 & 0.795 \\
$\mathrm{~T}$ & $0.30(0.08)$ & $61(14)$ & 0.32 & 0.045 \\
$\mathrm{~S}$ & $0.34(0.09)$ & $107(18)$ & 0.50 & 0.001 \\
$\mathrm{~N}$ & $0.30(0.07)$ & $68(10)$ & 0.27 & 0.091 \\
$\mathrm{I}$ & $0.38(0.10)$ & $118(18)$ & -0.20 & 0.223 \\
$p$-value & $<0.001$ & $<0.001$ & & \\
Glaucoma & & & & \\
$\mathrm{cp}$ & $0.24(0.10)$ & $66(19)$ & 0.63 & $<0.001$ \\
$\mathrm{~T}$ & $0.23(0.10)$ & $51(16)$ & 0.32 & 0.003 \\
$\mathrm{~S}$ & $0.23(0.14)$ & $74(29)$ & 0.72 & $<0.001$ \\
$\mathrm{~N}$ & $0.24(0.09)$ & $62(14)$ & 0.39 & $<0.001$ \\
$\mathrm{I}$ & $0.23(0.14)$ & $70(27)$ & 0.70 & $<0.001$ \\
$p$-value & 0.951 & $<0.001$ & & \\
\hline
\end{tabular}

All variables described as median (interquartile range); cp, circumpapillary; T, temporal; $\mathrm{S}$, superior; $\mathrm{N}$, nasal; I, inferior; $\rho$, spearman's correlation coefficient 


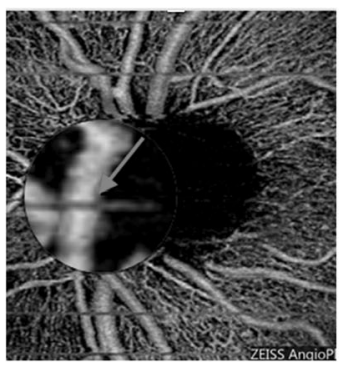

\begin{tabular}{|c|c|c|c|c|c|c|}
\hline & \multicolumn{2}{|c|}{ Movement } & \multicolumn{2}{|c|}{$\begin{array}{c}\text { ONH optical } \\
\text { hollow }\end{array}$} & \multicolumn{2}{|c|}{$\begin{array}{l}\text { Large vessel } \\
\text { crowding }\end{array}$} \\
\hline & $\begin{array}{c}\text { Yes } \\
(n=17)\end{array}$ & $\begin{array}{c}\text { No } \\
(n=23)\end{array}$ & $\begin{array}{l}\text { Small } \\
(n=28)\end{array}$ & $\begin{array}{c}B i g \\
(n=12)\end{array}$ & $\begin{array}{l}\text { Low } \\
(n=9)\end{array}$ & $\begin{array}{l}\text { High } \\
(n=31)\end{array}$ \\
\hline Median & 0.35 & 0.33 & 0.34 & 0.33 & 0.31 & 0.34 \\
\hline (IQR) & $(0.10)$ & $(0.06)$ & $(0.07)$ & $(0.07)$ & $(0.08)$ & $(0.07)$ \\
\hline$p$-value & \multicolumn{2}{|c|}{0.763} & \multicolumn{2}{|c|}{0.871} & \multicolumn{2}{|c|}{0.105} \\
\hline
\end{tabular}

Fig. 4 Influence of small movement artefacts, optic nerve head (ONH) optically hollow area and large vessel crowding within the healthy control group. The image shows minor movement artefacts (pointed by the red arrow), big ONH optically hollow area and large vessel crowding. Circumpapillary microvascular density (cpmVD), described as median, and interquartile range (IQR). were observed in all sectors. Moreover, when analysing mVD and RNFL sectors separately, a significant statistical difference between sectors was observed in the healthy group. Interestingly, such comparison in the glaucoma group was only significant for RNFL sectors, while mVD sectors showed no significant difference between them. The AROC for the cpRNFL thickness was 0.92 (0.87-0.97) and no significant statistical difference was observed when comparing to the cpmVD AROC $(p=0.15)$.

Potential sources of bias on cpmVD measurements are summarized in Fig. 4. No significant influence was detected $(p>0.05)$. Lastly, the median (IQR) fovea-disc axis angle for the healthy and the glaucoma groups was $6.6^{\circ}\left(4.5^{\circ}\right)$ and $7.4^{\circ}\left(6.9^{\circ}\right)$, respectively, where positive angle values represent an inferior foveal position in relation to the $\mathrm{ONH}$.

\section{Discussion}

A new procedure to automatically measure quantitative peripapillary microvasculature from angioOCT images was presented. By using it to compare healthy subjects with glaucoma patients, we observed higher cpmVD values in the first group. This follows what has been published by other authors [10,11]. Additionally, an evaluation of the $360^{\circ}$ profile of the microvascular density around the optic disc showed that healthy subjects had a higher variation of the cpmVD profile, with the typical 'double hump', with higher values in the superior and inferior sectors. On the other hand, the glaucoma subjects' profile was more uniform and 'flat', which is probably related to a greater damage in the superior and inferior sectors, leading to a more uniform microvascular density around the $\mathrm{ONH}$. As expected, the sectorial analysis showed that the largest differences between groups were found in the supero-temporal and inferior sectors, which coincides with the areas where glaucoma damage typically occurs [18] and the areas that had a greater amount of RNFL tissue in the healthy population.

Regarding the discriminating ability of mVD parameters, AROC values were comparable to those published by
Geyman et al. [19] and better than the values published by Yarmohammadi et al. [11], being the cpmVD, the parameter with the highest discriminating ability among the $\mathrm{mVD}$ parameters. In this work, we used a small ROI width (0.28 $\mathrm{mm}$ ) in order to have the same region analysed for all subjects avoiding the ONH optically hollow area. Nevertheless, the chosen width lead to an AROC (0.89), which shows how cpmVD can distinguish patients from controls. This AROC was comparable to those published in other papers such as by Geyman et al. (0.91) where a larger ROI with a $0.75 \mathrm{~mm}$ width was used. The obtained AROC for the mean RFNL thickness (0.93) was also similar to what has been published [11, 19] and no significant statistical difference was observed when comparing the AROCs of cpmVD and cpRFNL $(p=0.15)$.

Interestingly, in the glaucoma group, the $\mathrm{mVD}$ was similar between sectors $(p=0.951)$, while the RNFL thickness was higher in the superior and inferior sectors $(p<0.001)$. This finding could support the theory of vascular damage preceding structural damage. While the meaning of this finding remains elusive, it can either mean that the discriminating ability to detect the vascular information is higher than for RNFL measurements, but could also signal support to the theory that a wider vascular damage exists even in apparently preserved RNFL areas. However, prospective studies are necessary to reach such conclusion. So far, only two attempts have been done to use this technology for progression analysis. Shoji et al. [20] found a significant decrease in the macular vessel density of glaucoma patients with no significant changes in the ganglion cell complex thickness after 1 year of follow-up. On the other hand, Hollo [21] reported a 2-year evaluation of glaucoma patients that showed RNFL progression with no peripapillary vascular density changes. However, only mean circumpapillary values were used, which might have overlooked focal damage. In addition, both studies had small sample sizes $(n<33)$ and different vascular areas were studied. As such, further studies are needed to better understand the value of angioOCT in the detection of glaucoma progression. Dividing the studied area in sectors is 
relevant since most glaucoma cases initially present with a focal RGC loss and develop diffuse damage only at a later stage [22]. This might allow an earlier detection of damage that would be otherwise lost with a $360^{\circ}$ average. Also, regarding the fovea-disc axis angle, our study showed a wide variation, highlighting the importance of such a correction to perform sectorial comparisons between subjects. In addition to structure-structure correlation, our software is also built to allow a structure-function correlation (not shown in this manuscript), by dividing the ROI in the six sectors defined by Garway-Heath et al. [23]. Additionally, we tested if conditions that might introduce bias resulted in different cpmVD values. The algorithm proved to be robust even under conditions like movement artefact, different ONH dimensions and greater amount of large vessels (vessel crowding). Regarding the latter, a standard technique of measuring the local intensities $[11,24,25]$ is not accurate, since large vessels change between subjects and they are not the source of perfusion of the ONH. A few studies took into account the influence of vessel crowding by removing the large vessels from the original image based on thresholding techniques $[19,24,26]$. Such a procedure may provide a rough estimation of the microvascular density but gives rise to intra-operator variability since the threshold for the boundary between micro and macrovasculature is empirically established, which is subjective and will lead to differences between operators. On the other hand, the presented algorithm reduces the large vessel influence by employing a lower peak envelope technique, which is operator independent, and avoids the large intensity peaks generated by large volumetric blood flow by modulating their lower amplitude. Additionally, unlike the standard methods where binarization and thresholding are employed, the proposed technique does not require vessel removal from the main image, minimizing the bias added by imaging processing.

It must be pointed out that the glaucoma group had a mean visual field mean deviation of $-7.8 \mathrm{~dB}$, which makes it easier to distinguish from the normal group. Future studies will be done with less severe glaucoma cases, which will also look at how well this technique behaves for glaucoma severity detection. Additionally, by selecting only patients whose images had a quality equal or higher than 6 , we might be introducing a bias, towards less severe cases, with a better ocular surface/optical media. However, this is outside the scope of this study since our main goal was to describe a new methodology to measure circumpapillary microvascular density from angioOCT images. Also, we used a fixed inner and outer radius, which leads to measurements at a different distance from the ONH rim. This characteristic makes this software particularly useful for patient follow-up, where that distance will remain the same. Additionally, the outer radius was $1.44 \mathrm{~mm}$ instead of $1.5 \mathrm{~mm}$ to avoid the trademark symbols, which reduces the area of the ROI. The extra $0.06 \mathrm{~mm}$ represent a $21 \%$ increase of our current ROI, which could reduce the measurement variability and increase our ability to detect glaucomatous damage. Moreover, having $6 \times 6 \mathrm{~mm}$ scans would perhaps enable us to better detect capillaries, since part of them run alongside the large vessels at the area closest to the optic disc. However, only few patients were able to cooperate for good quality $6 \times 6 \mathrm{~mm}$ scans and we found that the $3 \times 3 \mathrm{~mm}$ scans already provided a good ability to detect glaucomatous damage. Finally, an automatic grading of image quality (rather than a subjective classification) would allow a more accurate assessment of potential sources of bias.

In summary, this open-source methodology can lead to a standardization of methods and thus allow a more accurate comparison of results among different research groups. In the future, we aim to improve it further, by introducing macular measurements and allowing the upload of images from other OCT devices.

\section{Summary}

\section{What was known before}

- Optical coherence tomography (OCT) angiography is a novel tool capable of distinguishing healthy controls from glaucoma patients. However, the current techniques to gather quantitative microvascular data have limited robustness or are not disclosed.

\section{What this study adds}

- We developed a novel open-source tool to automatically quantify the circumpapillary microvascular density from OCT angiography scans. Additionally, this tool corrects for the fovea-disc axis and avoids the influence of major retinal vessels. This tool can be used to compare results among different research groups.

\section{Compliance with ethical standards}

Conflict of interest The authors declare that they have no conflict of interest.

\section{References}

1. Tham YC, Li X, Wong TY, Quigley HA, Aung T, Cheng CY. Global prevalence of glaucoma and projections of glaucoma burden through 2040: a systematic review and meta-analysis. Ophthalmology. 2014;121:2081-90. 
2. Weinreb RN, Aung T, Medeiros FA. The pathophysiology and treatment of glaucoma: a review. JAMA. 2014;311:1901-11.

3. Sommer A, Tielsch JM, Katz J, Quigley HA, Gottsch JD, Javitt J, et al. Relationship between intraocular-pressure and primary open angle glaucoma among White and Black-Americans - the Baltimore Eye Survey. Arch Ophthalmol. 1991;109:1090-5.

4. Flammer J, Orgul S, Costa VP, Orzalesi N, Krieglstein GK, Serra LM, et al. The impact of ocular blood flow in glaucoma. Prog Retin Eye Res. 2002;21:359-93.

5. Tian K, Shibata-Germanos S, Pahlitzsch M, Cordeiro MF. Current perspective of neuroprotection and glaucoma. Clin Ophthalmol. 2015;9:2109-18.

6. Weinreb RN. Glaucoma neuroprotection: what is it? why is it needed? Can J Ophthalmol. 2007;42:396-8.

7. Abegao Pinto L, Willekens K, Van Keer K, Shibesh A, Molenberghs G, Vandewalle E, et al. Ocular blood flow in glaucoma the Leuven Eye Study. Acta Ophthalmol. 2016;94:592-8.

8. Rao HL, Kadambi SV, Weinreb RN, Puttaiah NK, Pradhan ZS, Rao DAS, et al. Diagnostic ability of peripapillary vessel density measurements of optical coherence tomography angiography in primary open-angle and angle-closure glaucoma. Br J Ophthalmol. 2017;101:1066-70.

9. Lee EJ, Lee KM, Lee SH, Kim TW. OCT angiography of the peripapillary retina in primary open-angle glaucoma. Invest Ophthalmol Vis Sci. 2016;57:6265-70.

10. Jia Y, Wei E, Wang X, Zhang X, Morrison JC, Parikh M, et al. Optical coherence tomography angiography of optic disc perfusion in glaucoma. Ophthalmology. 2014;121:1322-32.

11. Yarmohammadi A, Zangwill LM, Diniz-Filho A, Suh MH, Manalastas PI, Fatehee N, et al. Optical coherence tomography angiography vessel density in healthy, glaucoma suspect, and glaucoma eyes. Invest Ophthalmol Vis Sci. 2016;57:OCT451-9.

12. Chen CL, Bojikian KD, Wen JC, Zhang Q, Xin C, Mudumbai RC, et al. Peripapillary retinal nerve fiber layer vascular microcirculation in eyes with glaucoma and single-hemifield visual field loss. JAMA Ophthalmol. 2017;135:461-8.

13. Mammo Z, Heisler M, Balaratnasingam C, Lee S, Yu DY, Mackenzie P, et al. Quantitative optical coherence tomography angiography of radial peripapillary capillaries in glaucoma, glaucoma suspect, and normal eyes. Am J Ophthalmol. 2016;170: 41-9.

14. Akil H, Huang AS, Francis BA, Sadda SR, Chopra V. Retinal vessel density from optical coherence tomography angiography to differentiate early glaucoma, pre-perimetric glaucoma and normal eyes. PLoS ONE. 2017;12:e0170476.
15. Jampel HD. Target pressure in glaucoma therapy. J Glaucoma. 1997;6:133-8.

16. Mwanza JC, Lee G, Budenz DL. Effect of adjusting retinal nerve fiber layer profile to fovea-disc angle axis on the thickness and glaucoma diagnostic performance. Am J Ophthalmol. 2016;161: 12-21.e1-2.

17. Jesus DA, Barbosa BJ. 2018. https://drive.google.com/drive/ folders/1paQ6OYSq2HG_5a7HqUIDDakXWb76LwLh?usp=sha ring.

18. Mok KH, Lee VW, So KF. Retinal nerve fiber loss pattern in hightension glaucoma by optical coherence tomography. J Glaucoma. 2003;12:255-9.

19. Geyman LS, Garg RA, Suwan Y, Trivedi V, Krawitz BD, Mo S, et al. Peripapillary perfused capillary density in primary open-angle glaucoma across disease stage: an optical coherence tomography angiography study. Br J Ophthalmol. 2017;101: 1261-8.

20. Shoji T, Zangwill LM, Akagi T, Saunders LJ, Yarmohammadi A, Manalastas PIC, et al. Progressive macula vessel density loss in primary open-angle glaucoma: a longitudinal study. Am J Ophthalmol. 2017;182:107-17.

21. Holló, Gábor. Comparison of Peripapillary OCT Angiography Vessel Density and Retinal Nerve Fiber Layer Thickness Measurements for Their Ability to Detect Progression in Glaucoma. Journal of glaucoma 27.3.2018;302-305

22. Anton A, Yamagishi N, Zangwill L, Sample PA, Weinreb RN. Mapping structural to functional damage in glaucoma with standard automated perimetry and confocal scanning laser ophthalmoscopy. Am J Ophthalmol. 1998;125:436-46.

23. Garway-Heath DF, Poinoosawmy D, Fitzke FW, Hitchings RA. Mapping the visual field to the optic disc in normal tension glaucoma eyes. Ophthalmology. 2000;107:1809-15.

24. Akagi T, Iida Y, Nakanishi H, Terada N, Morooka S, Yamada H, et al. Microvascular density in glaucomatous eyes with hemifield visual field defects: an optical coherence tomography angiography study. Am J Ophthalmol. 2016;168:237-49.

25. Liu L, Jia YL, Takusagawa HL, Pechauer AD, Edmunds B, Lombardi L, et al. Optical coherence tomography angiography of the peripapillary retina in glaucoma. JAMA Ophthalmol. 2015; 133:1045-52.

26. Yarmohammadi A, Zangwill LM, Diniz-Filho A, Saunders LJ, Suh $\mathrm{MH}, \mathrm{Wu} \mathrm{Z}$, et al. Peripapillary and macular vessel density in patients with glaucoma and single-hemifield visual field defect. Ophthalmology. 2017;124:709-19. 